\title{
Transatlantica
}

Revue d'études américaines. American Studies Journal

\section{Seventies, Le choc de la photographie américaine}

Le jeu des 9 familles

\section{Sophie Hedtmann}

\section{(2) OpenEdition}

Journals

Édition électronique

URL : https://journals.openedition.org/transatlantica/4310

DOI : $10.4000 /$ transatlantica.4310

ISSN : $1765-2766$

Éditeur

Association française d'Etudes Américaines (AFEA)

Référence électronique

Sophie Hedtmann, «Seventies, Le choc de la photographie américaine », Transatlantica [En ligne], 1 |

2009, mis en ligne le 05 août 2009, consulté le 17 septembre 2021. URL : http://

journals.openedition.org/transatlantica/4310 ; DOI : https://doi.org/10.4000/transatlantica.4310

Ce document a été généré automatiquement le 17 septembre 2021.

\section{cc)}

Transatlantica - Revue d'études américaines est mise à disposition selon les termes de la licence

Creative Commons Attribution - Pas d'Utilisation Commerciale - Pas de Modification 4.0 International. 


\section{Seventies, Le choc de la} photographie américaine

Le jeu des 9 familles

Sophie Hedtmann

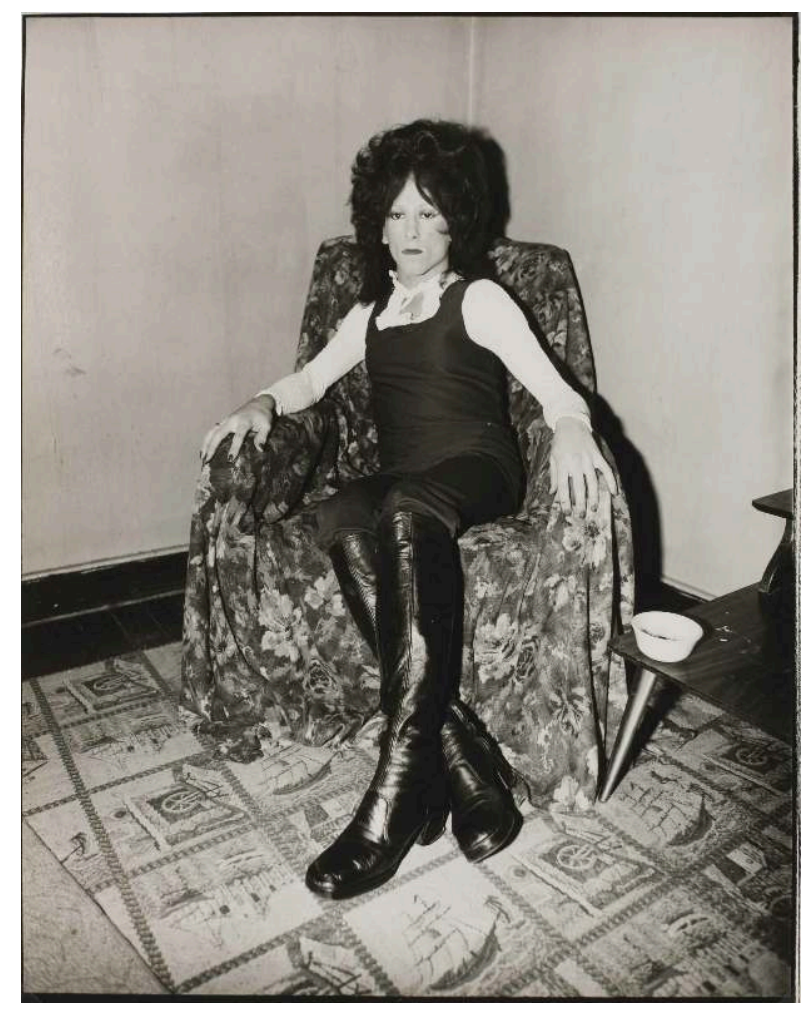


Jeffrey K. Silverthorne, Ugly Pat, 1972. BnF, dpt des Estampes et de la photographie, @ Jeffrey K. Silverthorne

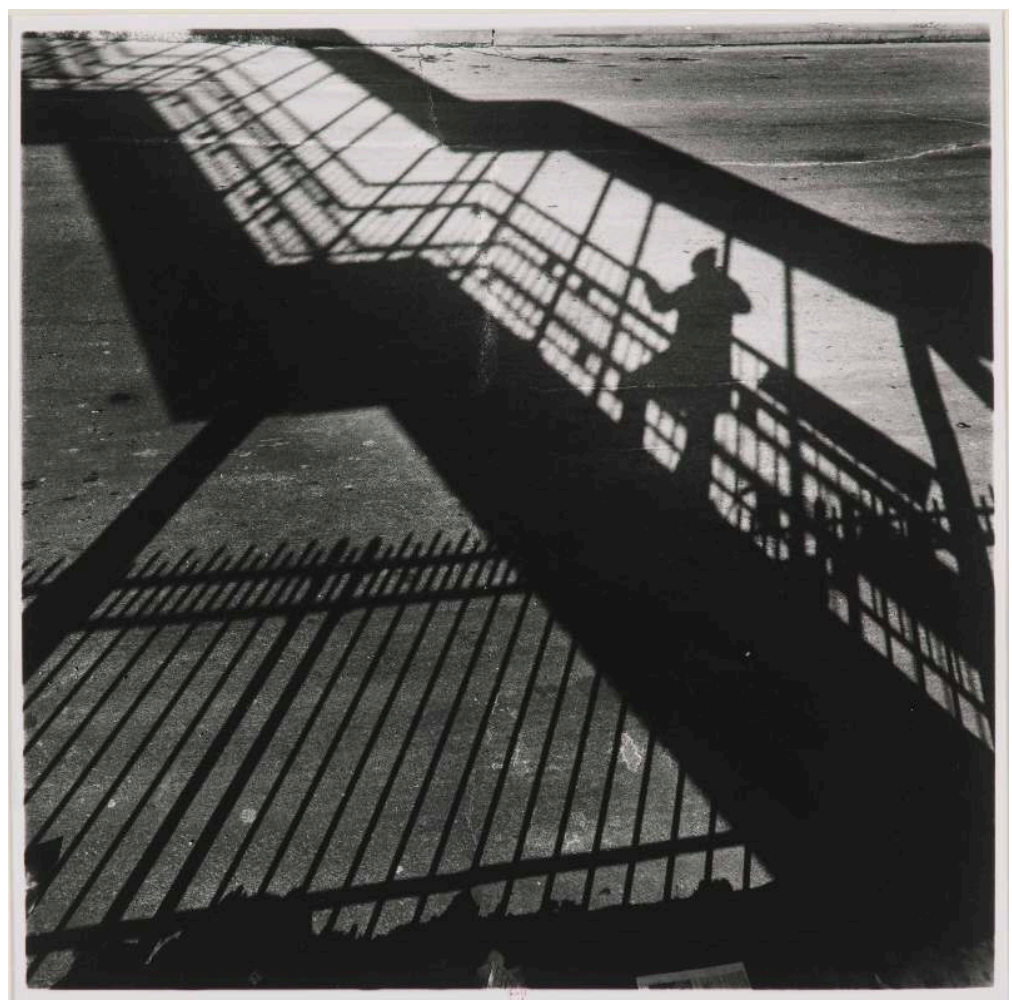

Arthur Tress, Série Shadow, 1975. BnF, dpt des Estampes et de la photographie $\odot$ Arthur Tress collection établie entre 1969 et 1996 par Jean-Claude Lemagny, alors conservateur du département photographique. L'exposition tente un exercice délicat: mettre en exergue les influences des regards. S'ensuit une sorte de ritournelle photographique, à découvrir comme si l'on surprenait une conversation d'images.

Biroleau, commissaire de l'exposition, a ainsi opté pour une approche formaliste plus qu'historique : «Il s'agit de favoriser le dialogue, la symbiose, la sympathie de ces images entre elles, d'esquisser une géographie des relations au sein de ce territoire, de comprendre les alliances et les alliages, les conflits dynamiques et les confluences créatrices ». En regroupant sous des thèmes divers plusieurs photographes, Anne Biroleau s'engage dans une présentation esthétisante de l'image. Davantage d'explications sur le contexte de l'époque auraient sans doute permis au visiteur de mieux appréhender les photographies. Néanmoins, le choix d'un parcours thématique permet de s'aventurer dans un jeu de l'esprit et de ressentir quel fut « le choc de la photographie américaine ", d'autant plus que nombre de ces images continuent à nous ébranler avec force. Comme l'écrit Anne Biroleau, la photographie "énonce quelques vérités sur la sensibilité et le rapport au monde de son auteur, et de même revêtant par là une qualité d'universalité, nous renvoie vers notre propre sensibilité et notre propre vision du monde. $»^{1}$. 
Imaginez dès lors un parcours, une sorte de "jeu des familles », qui se décline en neuf séquences : «Des précurseurs / Portraits / Les rues / Les mondanités; les gens ordinaires / Les marges / Géométrie ; Espace / Nature / Paysage minimal / Matière ; Forme / Le miroir obscur ». Certaines de ces séquences se définissent par leur sujet, « les mondanités » par exemple, et d'autres par leur forme comme "Géométrie ». Une seule se distingue, « le miroir obscur », comme venant confondre toutes les autres.

En choisissant ce parti, le spectateur est convié à comprendre le principe et la valeur intrinsèque du travail du photographe. La scénographie voulue par Anne Biroleau est construite à la manière d'un dispositif cinématographique, conçu comme un enchaînement de séquences.

6 Le visiteur est ainsi amené à découvrir une première "séquence» qui le plonge dans une avant-scène, celle des photographies de Walker Evans ${ }^{2}$ et Louis Faurer ${ }^{3}$. Cette salle consacrée aux « précurseurs » donne le ton sur lequel l'exposition s'engage. Pénétré de cet héritage, il plonge ensuite dans les diverses séquences proposées. La géographie du lieu - un long corridor - ne permet a priori pas cette organisation, mais suggère plutôt u enchaînement chronologique. Néanmoins, il ne faut pas hésiter à "sauter " des séquences afin de mieux comprendre où Anne Biroleau souhaite nous mener. Ainsi, une image de Louis Faurer fait apparaître une volonté de déconstruire le regard à travers une composition qui joue sur une esthétique surréaliste de reflets et d'ombres, l'environnement urbain étant perçu à travers les vitrines de la rue. Quelques cimaises plus loin, dans la séquence des "portraits », une impression de "déjà vu » se dégage d'un autoportrait de Lee Friedlander ${ }^{4}$ qui fait de son ombre sa signature. A travers «le miroir obscur » de la séquence suivante, Arthur Tress ${ }^{5}$ joue lui aussi avec les ombres qu'il fait émerger comme des fantômes dans des environnements urbains géométriques au point de ne plus être tout à fait humains. Au-delà des aspects formels, les séquences sont aussi là pour nous montrer comment des images pourraient entraîner dialogues et correspondances entre la solitude de personnages extravagants qui excitent souvent notre curiosité comme chez Mary Ellen Mark ${ }^{6}$ et les mondains des soirées de la jet set de Garry Winogrand ${ }^{7}$. Chez l'une, un personnage est assis, lointain, indifférent au regard du photographe, chez l'autre, les personnages sont figés dans leurs attitudes, presque irréels, mais tout aussi étrangers à celui qui les fixe.

7 Si les formes traduisent la volonté de "dire ", le bouleversement créé à la vue de ces images par le spectateur provient d'une véritable appropriation de l'outil photographique par le photographe. En choisissant comme sujet le corps recousu d'une jeune femme à la morgue, Jeffrey K. Silverthorne ${ }^{8}$ opte pour un cadrage serré : loin de s'effacer, il dit au contraire sa fascination. De même, Larry Clark ${ }^{9}$ semble s'immiscer dans la sexualité des adolescents en s'emparant de leur image, sans recul, abruptement.

8 Ce que l'exposition révèle aussi, c'est aussi une crudité extrême du regard. Les photographes cherchent à montrer ce que personne alors ne voulait voir : une société en déliquescence, des paysages urbains déshumanisés, à la beauté dérangeante. Ils osent désormais utiliser ce médium pour dévoiler leurs mondes intérieurs.

9 L'exposition " 70', le choc de la photographie américaine » est ainsi une série de « chocs » visuels qui définissent la nature même de la photographie et semblent tous se rapporter aux mots de Walker Evans: «les images parlent d'elles-mêmes, silencieusement, visuellement, ou bien c'est un échec $»^{10}$. 


\section{NOTES}

1. Biroleau, Anne, «L'évidence de la case vide», 70'. Le choc de la photographie américaine, Anne Biroleau, Gilles Mora, Paris, BnF, 2008, p. 25

2. http://images.google.fr/images?

$\mathrm{hl}=$ fr\&q=Walker+Evans\&um=1\&ie=UTF-8\&sa=X\&oi=image_result_group\&resnum=1\&ct=title

3. http://www.agathegaillard.com/Faurer.html

4.

http://images.google.fr/images?

um=1\&hl=fr\&q=Lee+Friedlander\&btnG=Recherche+d\%27images

5. http://images.google.fr/images?um $=1 \&$ hl=fr\&q=Arthur+Tress\&btnG=Recherche $+\mathrm{d} \% 27$ images

6. http://www.maryellenmark.com/

7. http://www.mocp.org/collections/permanent/winogrand_garry.php http://www.moma.org/collection/browse_results.php?

criteria=0\%3AAD\%3AE\%3A6399\&page_number=1\&template_id=6\&sort_order=1

8.

http://images.google.fr/images?um=1\&hl=fr\&q=Jeffrey+K.

+Silverthorne+\&btnG=Recherche+d\%27images

9. http://www.larryclarkofficialwebsite.com/

10. "For the thousandth time, it must be said that pictures speak for themselves, wordlessly, visually, or they fail. » Cité dans Anne, Biroleau, «L'évidence de la case vide », 70'. Le choc de la photographie américaine, Anne Biroleau, Gilles Mora, Paris, BnF, 2008, p. 24

\section{INDEX}

Thèmes : Trans'Arts 